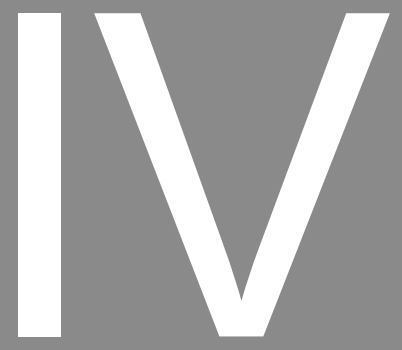

\title{
GRADO DE ACEPTACIÓN DE UN MODELO DE EVALUACIÓN DEL APRENDIZAJE BASADO EN COMPETENCIAS
}

\section{Degree of acceptance of a learning assessment model based on competencies \\ Universidad Peruana Unión, Perú}

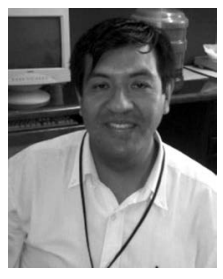

\section{Moisés Díaz Pinedo}

Licenciado en Educación por la Universidad Peruana Unión. Magíster en Educación con mendición en Investigación y Docencia Universitaria por la Universidad Peruana Unión. Doctor en Educación con mención en Diseño Curricular e Instrucción. Miembro de REDDOLAC - Red de docentes de América Latina y del Caribe. Docente principal de la Universidad Peruana Unión. Asimismo, realiza actividades de docencia en diversos posgrados organizados por dicha universidad. 


\section{Resumen}

En el presente artículo se consideran la importancia que tienen los modelos de evaluación basados en competencias. En primera instancia se encuentran los antecedentes que llevaron a su realización, como preámbulo al problema que se resuelve. Asimismo, se considera el grado de aceptación del modelo basado en competencias. Seguidamente, se presentan los procesos metodológicos que sirvieron para proporcionar una respuesta empírica y su concordancia con la teoría. Entre las conclusiones se encontró que el modelo de evaluación del aprendizaje muestra niveles de aceptación (75.25\%). Asimismos, se recomienda configurar un sistema de evaluación bajo un modelo como el presente, donde se integren la actuación, el desempeño, los alcances, las metas y unos logros claros y bien definidos; que, además, sea fiable y permita la identificación del nivel de eficiencia con que se realizan las actividades individuales y grupales.

Palabras clave: Competencias, evaluación, estrategias de aprendizaje, modelo.

\section{Abstract}

In this article we consider the importance of evaluation models based on competencies. First, we find the background that led to its realization, as a preamble to the problem is solved. It also considers the degree of acceptance of competencybased model. Then, we present the methodological processes that were used to provide an empirical answer and its agreement with the theory. Among the findings it was found that the learning assessment model sample acceptance levels (75.25\%). It also is recommended to set an evaluation system under a model such as this, which integrates performance, performance, scope, goals and achievement clear and well defined, that is also reliable and allow the identification of the level of efficiency are performed individual and group activities.

Key words: Skills, evaluation, learning strategies, model. 


\section{Introducción}

El interés creciente por las competencias educativas y su repercusión en la evaluación de las competencias es consecuencia de la influencia de su utilización en el mercado laboral, pero de forma más concreta de las evaluaciones realizadas por la Internacional Association for Educational Achievement (IEA) de Estados Unidos y de las evaluaciones en la OCDE (PISA, por sus siglas en inglés). El resultado ha sido la generalización del currículo por competencias con sus sistemas de evaluación, tanto en la educación obligatoria, como en la educación superior, así como en la formación permanente.

Actualmente, el Instituto CIFE (Centro de Investigación en Formación y Evaluación) de Colombia, en base a diversos procesos de investigación que lleva a cabo en diferentes países de América Latina, ha logrado sistematizar o generar una metodología pertinente de valoración de las competencias en educación, siguiendo el programa VALORA que se caracteriza porque aborda la evaluación con un enfoque formativo, buscando el desarrollo continuo de las potencias y talentos de los estudiantes, para lo cual tiene como base la metacognición, el proyecto ético de vida, el trabajo con proyectos integradores y el aprender a actuar con idoneidad ante los problemas de la vida. Asimismo, aborda la evaluación de las competencias como una propuesta educativa que tiene en cuenta los retos actuales y futuros del contexto. Sin embargo, también considera los elementos más relevantes y pertinentes de la evaluación tradicional, buscando la articulación de los procesos cualitativos con los aspectos cuantitativos en el aprendizaje. Para ello analizan los niveles de desempeño o de dominio que tienen los estudiantes, y con base en esto establecen las calificaciones numéricas (si es necesario) como una representación de dichos niveles de desempeño. En su propuesta determinaron los siguientes ejes: (a) formar y aplicar la competencia docente de evaluación, (b) establecer el fin de la evaluación de las competencias, (c) enfocarse en aprendizajes esperados y evidencias, (d) emplear problemas del contexto en la evaluación, (e) evaluar por medio del portafolio, (f) planear y utilizar mapas de aprendizaje, (g) diseñar y aplicar instrumentos de evaluación basados en problemas, (h) aplicar la metacognición, (i) realimentar a los estudiantes con asertividad (Tobón, 2011).

Pimienta (2008), presenta una somera revisión de distintos modelos de evaluación como primer antecedente al estudio de la evaluación del aprendizaje basado en competencias, considerando el desarrollo histórico de la educación basada en competencias. Así tenemos los siguientes: (a) modelo tyleriano, (b) modelo científico de Suchman, (c) modelo orientado a la planeación de Cronbach, (d) modelo CIPP de Stufflebeam, (e) modelo centrado en el 
cliente de Stake, (f) modelo iluminativo y holístico, (g) modelo centrado en el consumidor de Michael Scriven, y (h) modelo sistémico de Arturo de la Orden Hoz.

Tobón et al. (2010), manifiestan que desde la década de los noventa, el modelo de competencias en educación, y por ende la evaluación, se han consolidado debido al aporte de los diversos enfoques o perspectivas de las competencias que enfatizan determinados aspectos y tienen visiones diferentes. Estos enfoques son: funcionalista, conductual-organizacional, constructivista y socioformativo.

Así surge un nuevo modelo de evaluación que, a finales de la década de los noventa y comienzos del 2000, empezó a estructurarse bajo el enfoque socioformativo, el cual también se suele denominar enfoque sistémico complejo o enfoque complejo. Este modelo concibe la formación de las competencias como parte de la formación humana integral, a partir del proyecto ético de vida de cada persona, dentro de escenarios educativos colaborativos y articulados con lo social, lo económico, lo político, lo cultural, el arte, la ciencia y la tecnología (Tobón et al., 2010).

Considerando la información anterior, siendo necesario que haya cambios pertinentes en la evaluación de las competencias en todos sus planteamientos, objeto, agentes, momentos, así como en los instrumentos y técnicas que posibiliten su puesta en práctica, y en vista de la implementación del Modelo Educativo 2010, es necesario complementarlo con un Modelo de Evaluación del Aprendizaje Basado en Competencias. Por tanto, el problema de investigación se declara mediante la siguiente pregunta:

De los elementos básicos (principios, procesos, estrategias) que son considerados para la estructuración de un modelo de evaluación del aprendizaje basado en competencias, ¿cuál es el grado de aceptación de dicho modelo entre administradores y docentes de la UM durante el primer semestre del 2012 ?

\section{Metodología}

La metodología se ubica dentro del tipo cuantitativo, cualitativo, descriptivo, de encuesta, exploratorio, de campo y de corte transversal. Se midieron las variables de la manera más precisa posible, utilizando sus valoraciones a fin de determinar el grado de aceptación del modelo teórico de evaluación del aprendizaje basado en competencias y aplicando el instrumento una sola vez.

Se administraron los instrumentos a 451 estudiantes y 164 administradores y docentes de las ocho facultades y escuelas. En su mayoría fueron varones, incluyendo estudiantes de dos grados (segundo y cuarto semestres) 
que están en el plan del Modelo 2010, con un promedio de edad de 19 años para los estudiantes y de 45 años para los administradores y docentes. Se administraron los instrumentos de manera personal, durante sus períodos de clase para los estudiantes en los meses de abril y mayo de 2012. Y para los administradores y docentes durante sus períodos de descanso en sus cubículos personales durante el mes de junio del mismo año.

Los instrumentos utilizados fueron dos. Uno orientado a los alumnos y otro a los administradores y docentes. El instrumento para estudiantes consta de 13 declaraciones, a las que se agregan los datos demográficos y preguntas abiertas sobre estrategias utilizadas por los docentes y experiencias vividas en cuanto a la evaluación del aprendizaje basado en competencias, haciendo un total de 20 elementos. Mientras que el instrumento para administradores y docentes consta de 61 declaraciones, a las que se agregan los datos demográficos y preguntas abiertas sobre creencias y experiencias en la práctica de la evaluación, haciendo un total de 73 elementos. Las declaraciones fueron valoradas en una escala de 1 a 5 .

Para los análisis estadísticos se utilizaron la estadística descriptiva como medida de tendencia central y dispersión, así como tablas y gráficos; la prueba t de Student para muestras independientes; la prueba estadística de Kruskal-Wallis (prueba no paramétrica) para muestras independientes; la prueba de Regresión Lineal Múltiple; el análisis categórico de las respuestas que ayudaron a comprender el fenómeno estudiado. Para ello utilizamos la técnica de análisis de contenido, en donde la agrupación semántica juega un papel importante para la categorización de las ideas en torno del asunto en cuestión; y el modelo de ecuaciones estructurales (SEM), tomando en consideración tres criterios de bondad de ajuste (Hancok y Mueller, 2004; Hair et al., 1999). Se utilizó el estadístico Chi cuadrada como medida de ajuste total con un nivel de significatividad mayor a .05. El ajuste de parsimonia se valoró mediante el índice RMSEA, con un nivel de aceptación menor a .08. Por último, el CFI sirvió para medir el ajuste comparativo, aceptándolo cuando adquiriese valores mayores a .9. En todos los análisis se utilizaron el paquete estadístico Statistical Package for the Social Sciences (SPSS, versión 17.0 para Windows) y el AMOS 6.0.

En síntesis, se utilizaron los análisis descriptivos, categóricos, de validez y confiabilidad y análisis exploratorio. Para el análisis exploratorio se utilizaron los métodos modelo de ecuaciones estructurales (SEM), ANOVA y prueba t de Student. 


\section{Resultados}

Para determinar la validez y confiabilidad de los instrumentos, utilizamos los procedimientos siguientes: (a) comprobar si las escalas cumplen las condiciones necesarias, a través de la prueba de esfericidad de Bartlett y el valor KMO; (b) aplicar el análisis factorial, utilizando la técnica de análisis de componentes principales con rotación Varimax y (c) confiabilidad, a través del coeficiente $\alpha$ de Cronbach. Los resultados fueron, tanto para la escala de administradores y docentes como para los estudiantes, los siguientes: la prueba de esfericidad de Bartlett, significativa para todas las variables; el valor KMO, regular en algunos factores y sobresaliente en otros.

Con la aplicación de la técnica de análisis de componentes principales con rotación Varimax para extraer los factores, se obtuvo lo siguiente:

En el CEVACOM se formaron cuatro agrupaciones de las 18 declaraciones correspondientes, en lo siguiente: evaluación orientada hacia los contenidos, evaluación orientada hacia lo laboral, evaluación orientada hacia la persona y evaluación orientada hacia lo significativo, explicando el 51.81\% de la varianza total de la matriz de correlaciones. La fiabilidad total de la subescala, medida por el Alfa de Cronbach, obtuvo un valor de .791, considerado aceptable.

Para el GaMoTeC se procedió de la misma manera que el primero, solo que la extracción de factores se hizo con cada uno de sus elementos. Así, en el elemento Principios se formaron dos factores, explicando el $56.20 \%$ de la varianza total de la matriz de correlaciones, con una confiabilidad elevada (.817); en el elemento Procesos se retuvo un solo factor, el cual explica el $50.98 \%$ de la varianza total de la matriz de correlaciones, con una confiabilidad elevada (.860); y para el elemento Estrategias, se formaron tres factores, explicando el $53.91 \%$ de la varianza total de la matriz de correlaciones, con una confiabilidad elevada (.825). La confiabilidad total de la subescala, medida por el $\alpha$ de Cronbach, fue elevada (.930).

Asimismo, para el constructo GEAEV, se siguieron los mismos procedimientos anteriores. Los factores agrupados fueron tres, explicando el 59.24\% de la varianza total de la matriz de correlaciones. El $\alpha$ de Cronbach para toda la subescala fue de .800 , considerado elevado.

En el constructo GEAEV, correspondiente a la escala de los estudiantes, se agruparon dos factores, explicando el $52.37 \%$ de la varianza total de la matriz de correlaciones. El coeficiente $\alpha$ de Cronbach fue de .796, considerado aceptable.

El siguiente resultado a considerar es el comportamiento de las variables. Se observó que las características de la variable conceptualización 
orientada hacia los contenidos es significativo y tiene un comportamiento normal que no excede el valor crítico, mientras que los que se orientan hacia lo laboral y hacia la persona exceden de acuerdo al valor crítico en simetría pero no en curtosis. Los valores generales del CEVACOM (Simetría $=0.796$; Curtosis $=0.369$ ) indican que su comportamiento es normal.

En las características de la variable GaMoTeC, casi todas exceden en sus valores, excepto el elemento Principios que fundamenta la evaluación de las competencias. La variable GaMoTeC es no normal en lo que se refiere a las características PriOPR, ECPRO, EstCPA, EstACH y EstCC.

Las características de la variable GEAEV, a nivel de docentes, todas tienen un comportamiento normal, no exceden el valor crítico. Mientras que las características de la variable GEAEV, a nivel de estudiantes, una excede el valor crítico en simetría y es negativo, mas no en curtosis. La característica Gealn muestra un comportamiento normal.

Los datos demográficos revelan que en el estudio participaron 451 estudiantes, distribuidos en ocho facultades y diecinueve escuelas, de los cuales un $48.3 \%$ son mujeres y un $51.7 \%$ hombres, cuyas edades fluctúan entre los 16 a los 46 años. La edad predominante es de 19 años, representando el $26.6 \%$ del total de la muestra. Al momento de aplicar el instrumento, el 53.4\% estudiaban el primer año de su carrera (segundo semestre) y el $46.6 \%$ el segundo año (cuarto semestre). Todos son del plan 2010. La facultad más representativa es la de Ciencias de la Salud, con una frecuencia de 244, el cual representa el $54.1 \%$ del total de la muestra, y dentro de ella la Escuela de Medicina con una frecuencia de 120 , lo que representa el $26.6 \%$.

Asimismo, los administradores y docentes participantes en el estudio fueron 164 . El $17.1 \%$ son administradores y el $82.9 \%$ docentes, de los cuales un $39.6 \%$ son mujeres y un $60.4 \%$ hombres, cuyas edades fluctúan entre los 23 y los 69 años. La edad predominante es de 45 años, representando el $7.3 \%$ del total de la muestra. En su mayoría tienen el nivel académico de posgrado, siendo el $62.8 \%$ del nivel de maestría y el $18.3 \%$ del nivel de doctorado. La facultad más representativa es la de Ciencias de la Salud con una participación de 50 docentes, lo cual representa el $31.4 \%$ del total de la muestra. Y entre las escuelas, la de Educación con una participación de 23 docentes que representan el $14.5 \%$ del total de la muestra. El tiempo de servicio a la institución fluctúa entre 1 a 40 años, y el tiempo que han estado en el puesto que ocupan está entre 1 a 39 años, siendo la media de 14.59 y 10.59 años, respectivamente.

El grado de aceptación del modelo teórico de evaluación del aprendizaje basado en competencias (GaMoTeC) fue de 4.01 en una escala de 1 a 5 . Esto indica que los docentes y administradores de la UM están de acuerdo con 
el modelo teórico y lo aceptan en un 75.25\%. La desviación típica correspondió a 0.494 , indicando una opinión bastante homogénea.

En cuanto a la conceptualización de la evaluación del aprendizaje basado en competencias, el $76 \%$ de los docentes considera que la evaluación debe orientarse hacia lo laboral. Un $73.75 \%$ hacia lo significativo, un $67.75 \%$ hacia la persona y un $59.75 \%$ hacia los contenidos.

En cuanto a la variable GEAEV, para los docentes el grado de estimulación del aprendizaje a través de las evaluaciones es alto $(M=4.05, D E=.391)$. Es decir, las competencias en general se estimulan en un $81.75 \%$, las actitudes positivas en un $80.75 \%$ y las actitudes negativas en un $49.00 \%$. Mientras que para los alumnos es media unidad más baja $(M=3.54, D E=.509)$. El $66.50 \%$ de los alumnos afirma que les trae mucho beneficio y a un $53.25 \%$ les da cierta inseguridad.

Con respecto a las creencias sobre las prácticas de evaluación por competencias, 125 administradores y docentes, que representan el $76.2 \%$, respondieron que sí creen que se están evaluando las competencias en la UM, y $39(23.8 \%)$ respondieron que no creen. Al mismo tiempo, dieron 93 razones (72.09\%) por el sí, y 36 razones (27.91\%) por el no.

En cuanto a las estrategias, 124 administradores y docentes (75.6\%) respondieron que sí creen que las estrategias que se usan en la evaluación de las competencias en la UM son las adecuadas y 40 (24.4\%) respondieron que no. Se expresaron 122 razones, de las cuales 91 (74.59\%) apoyan el sí y 31 (25.41\%), el no.

En los procesos que apoyan el desarrollo de las competencias, 131 (79.9\%) respondieron que sí creen que los procesos que se siguen en la evaluación de las competencias en la UM están apoyando al desarrollo de las competencias y 33 (20.1\%) respondieron que no. Los que respondieron que sí se fundamentan en 71 razones (71.72\%) y los que respondieron que no expresaron 28 razones (28.28\%).

Sobre los principios filosóficos, 156 administradores y docentes respondieron que sí creen que las evaluaciones de las competencias en la UM toman en cuenta los principios filosóficos de la educación adventista. Esto representa el $95.1 \%$ y ocho respondieron que no, representando al $4.9 \%$ de los administradores y docentes. Sus razones fueron 89 para el sí (88.12\%) y 12 para el no (11.88\%).

Asimismo, los administradores y docentes escribieron 86 sugerencias sobre otros elementos que podrían ser útiles en el modelo de evaluación del aprendizaje basado en competencias. Estas se agruparon en trece áreas: (a) nuevas estrategias, (b) procesos de evaluación, (c) prácticas profesionales, (d) reafirmación del modelo, (e) competencias bien definidas, (f) apoyo comuni- 
tario, (g) capacitaciones, (h) involucramiento maestro-alumno, (i) actitudes del docente, (j) investigación, (k) principios básicos, (I) docente no capacitado y (m) otras observaciones.

También, escribieron 106 sugerencias de cómo se deberían evaluar las competencias de los alumnos. Dichas sugerencias fueron clasificadas en ocho áreas: (a) emplear problemas del contexto en la evaluación, (b) enfocarse en aprendizajes esperados y evidencias, (c) tener claro el propósito de la evaluación, (d) trabajar o actuar de acuerdo al modelo, (e) diseñar y aplicar instrumentos de evaluación, (f) formar y aplicar la competencia docente de evaluación, (g) evaluar por medio del portafolio y (h) realimentar a los estudiantes con asertividad.

El $82.7 \%$ de los estudiantes manifestaron que la estrategia de evaluación que mayormente utilizan los docentes de la UM en sus clases es el portafolio; el 77.2\% aseveraron que le sigue la investigación; el 73.2\% identificaron los proyectos y el $68.1 \%$ se refirió a la resolución de problemas.

Asimismo, el $94.5 \%$ manifestaron que la estrategia que menos usan son los registros de incidentes críticos. Le siguen las escalas de valoración (92\%), las listas de control (86.5\%), los debates (83.4\%), la evaluación por pares o coevaluación (79.2\%) y las pruebas de desarrollo (71.6\%).

Los estudiantes escribieron 361 experiencias sobre las evaluaciones realizadas en el aula, las cuales fueron categorizadas en dos grandes grupos: experiencias positivas, destacándose la satisfacción con el modelo de evaluación y la estimulación del aprendizaje; y experiencias negativas, en cuanto a conductas éticas en la evaluación. Asimismo, manifestaron 30 recomendaciones entre las que se destacan la practicidad de las evaluaciones y la capacitación a los alumnos en este modelo, tomándolo en cuenta.

Los resultados de la relación entre las variables CEVACOM y GaMoTeC alcanzaron un nivel de significatividad aceptable y medianamente fuerte. Siendo los factores que más aportan para la relación, la evaluación orientada hacia la persona y la evaluación orientada hacia lo significativo, y la que menos aporta, la evaluación orientada hacia los contenidos (factores del CEVACOM). Mientras para la variable GaMoTeC, las que más aportan fueron: el elemento procesos y el elemento principios, siendo el elemento estrategias el que menos aporta en la relación, aunque existe equilibrio en los aportes.

Según el análisis del SEM, se encontró una bondad de ajuste alta en el CFI (.977), aceptable en el RMSEA (.073) y significativa con respecto al ajuste global, según la Chi-cuadrada.

Se observó que sí existe diferencia entre las medias de la variable GEAEV, según la percepción de docentes y estudiantes. La percepción de los docentes sobre el grado de estimulación del aprendizaje a través de la evalua- 
ción en sus alumnos es mayor $(M=4.05)$ mientras que la percepción del estudiante es poco menor $(M=3.54)$. Asimismo, las trece declaraciones muestran el $p$-valor asociado igual a 0.000 , indicando la existencia de diferencias entre las medias de cada declaración.

También se observó que existen diferencias significativas en la variable GaMoTeC según la facultad a la que pertenece el docente. Y considerando sus elementos estructurantes, encontramos diferencias significativas en Principios $\left(X^{2}(8)=17.258, p=.028\right)$ y Estrategias $\left(X^{2}(8)=17.837, p=.022\right)$. En el elemento procesos la diferencia no fue significativa.

Observando los rangos, la que más acepta el modelo es la escuela de Artes y Comunicación (rango = 112.46); le sigue la facultad de Educación (rango $=95.48$ ) y en tercer lugar, Música (rango $=89.75$ ). La Facultad de Ingeniería y Tecnología fue la que menos aceptó el modelo (rango = 53.54).

Por último, a través de la prueba estadística de Regresión Lineal, se determinó la medida en que las dimensiones del GaMoTeC predicen el GEAEV, observando que el GaMoTeC explica el $28.6 \%$ de la variación de GEAEV, siendo la dimensión Principios que orientan los procesos y resultados de la evaluación (PriOPR) y la dimensión Estrategias que fomentan los contenidos conceptuales (EstCC) la que explica de manera significativa la variable GEAEV. Asimismo, el modelo entre las variables es significativo $(F=33.58, g l .=161, p=.000)$.

\section{Discusión}

En esta sección se discuten los resultados que dan respuesta a las preguntas de investigación, centrados específicamente en tres áreas bien definidas: Grado de aceptación del modelo (GaMoTeC), conceptualización de la evaluación de las competencias (CEVACOM) y grado de estimulación del aprendizaje (GEAEV).

Sobre el GaMoTeC, los resultados indican una aceptación del modelo en un $75.25 \%$, siendo la opinión de los administradores y docentes de la UM homogénea en cuanto a los elementos que conforman el modelo; es decir, están de acuerdo que los principios, procesos y estrategias sean los elementos básicos del modelo. Asimismo, las facultades y escuelas que más aceptan el modelo son aquellas que se orientan hacia las ciencias humanas: Artes y Comunicación, Educación y Música; mientras la que menos acepta es la Facultad de Ingeniería y Tecnología, mostrando que existen diferencias significativas, según el análisis de los estadísticos de contraste, en Principios y Estrategias.

Las interrogantes son: ¿por qué la aceptación es un $75.25 \%$ y no un $90 \%$ o $100 \%$ ? ¿A qué se debe que la aceptación por facultades y escuelas se orienta más a las ciencias humanas y no a las ciencias tecnológicas? 
La respuesta a estas interrogantes se podría dar con la misma opinión de los docentes de la UM, cuando manifiestan que "se está iniciando, falta experiencia. Es una cuestión de tiempo"; o como estas: "algunos las están implementando, otros no sabemos cómo implementarlas en las materias que impartimos"; "los maestros siguen con las mismas prácticas escolares antiguas"; "muchos profesores siguen haciendo lo mismo y de la misma manera de siempre"; "falta compromiso del docente, no cree en este plan. La formación profesional que tuvimos no involucraba competencias"; "el maestro sigue haciendo una labor solitaria, casi no hay trabajo colaborativo entre docentes" y, por último, "es la moda, pero no se sabe qué son".

A través de las declaraciones se perciben que son muchos los factores que podrían haber influido para que el modelo tenga una aceptación "medianamente aceptable": tendencias, innovación reciente, modelo tradicional, complejidad, resistencia al cambio, características del personal, cosmovisión, cultura, entre otras.

Diversos autores manifiestan que, si bien es cierto que una de las tendencias es la formación basada en competencias (Tobón, 2010), la preocupación por la pertinencia, eficiencia y calidad de las instituciones universitarias tiene su relación con las competencias de los alumnos, atribuyéndole importancia por sus posibilidades de transferencia y aplicación para ejercer una profesión (Quiroz, 2010). Y agrega que el modelo de evaluación de las competencias aparece como una opción para resolver las demandas de la sociedad del conocimiento y obliga a las universidades a replantear lo que comúnmente han considerado como formación. Ruiz (1999), manifiesta que la instauración de los modelos de evaluación basados en competencias y llevados a cabo en un ambiente complejo, los nuevos planteamientos, se oponen a los esquemas conceptuales tradicionales de los docentes. Por tanto, es preciso cambiar la orientación de la educación superior, homogeneizar el sistema evaluativo y nivelarlo de acuerdo a las tendencias, definiendo los contenidos del proceso enseñanza-aprendizaje en el cual se involucra la evaluación.

Coronado, Sánchez, Vega y León y Jiménez (2001), expresan que hay que definir modelos de evaluación que permitan reestructurar y operar los avances en el ámbito educativo, porque el mundo real requiere entrelazar la actividad intelectual con la manual y laboral, en busca del saber hacer. Moreno (2000), agrega que el modelo de evaluación basado en competencias es una posibilidad para tal fin.

Reynaga (2003), asevera que las experiencias en competencias en el nivel superior son incipientes. Lluch (1998), dice que se espera que con su inclusión (del modelo de evaluación) se promueva una experiencia práctica. Al inicio de esta discusión se dijo que los elementos que conforman el modelo 
por tener medias altas ( $>4$ ) en la opinión de los administradores y docentes era homogénea y, por tanto, existe aceptación de dichos elementos, aunque la mayor aceptación se da en los elementos principios y procesos y, en menor proporción, en estrategias. Clark (2000), al respecto dice que el núcleo académico altamente estimulado aceptará o se opondrá a una transformación significativa. Es aquí, en las distintas unidades del núcleo académico, donde es más probable que los cambios propuestos y las innovaciones fallen. Si las unidades básicas se oponen o ignoran las posibles innovaciones, la vida de las instituciones proseguirá en gran medida como antes. El núcleo académico es donde los valores académicos tradicionales están más arraigados. La combinación requerida de esos valores con los nuevos puntos de vista de gestión tendrá que trabajar en este nivel.

Es por esto que para identificar la aplicación de los elementos del modelo de evaluación de las competencias en la UM, se recurre a sus actores. Un docente expresó: "los elementos del sistema de evaluación actual me parecen suficientes, lo que faltaría es sistematizar el modelo".

¿Por qué el elemento estrategias tiene una menor proporción de aceptación? Por el mismo hecho de que a las estrategias de evaluación tradicionales de un momento a otro, y sin ninguna reflexión y transformación, con frecuencia se les denomina estrategias de evaluación de competencias sin serlo en realidad, porque se siguen enfocando en contenidos u objetivos (no tienen en cuenta los principios esenciales de las competencias). Por ejemplo, muchas publicaciones abordan las rúbricas para evaluar competencias, pero se quedan en la aplicación de tales rúbricas en contenidos sin problemas contextuales y sin considerar niveles de desempeño. De ahí la necesidad de diseñar estrategias precisas de evaluación de competencias basados en los niveles de desempeño, los problemas del contexto y los procesos de autoevaluación, coevaluación y heteroevaluación (Tobón, 2011). Esta declaración confirma lo que los docentes de la UM expresaron: "no todos saben evaluar o conocen estrategias de evaluación por competencias"; "todavía veo algunas estrategias que solo evalúan contenido".

En relación a la aceptación del modelo por facultades y escuelas, la interrogante del por qué aquellas que se orientan hacia las ciencias humanas, artes y comunicación, educación y música son las que más fácilmente asimilaron el modelo; mientras que a las facultades que se relacionan con la tecnología, el rigor científico, les cuesta aceptar el cambio. La respuesta a esta interrogante se centra en las características específicas de cada profesión. En la Escuela de Artes y Comunicación siempre estuvieron familiarizados con el uso de los portafolios, que en la actualidad es una estrategia innovadora de evaluación de las competencias. Danielson y Abrutyn (2002), mencionan que 
los portafolios no son una invención reciente. El concepto existe desde hace mucho en numerosos ámbitos fuera del aula. Artistas, arquitectos y fotógrafos los usan para presentar sus trabajos a clientes potenciales. En general se admite que los portafolios de arte son la manera óptima de abarcar lo mejor del desempeño del estudiante en las artes, con dibujos, diapositivas y ejemplos de música compuesta y ejecutada; quiere decir que los docentes están habituados a trabajar de esta manera; $y$, a partir de la información proporcionada por los docentes de la Escuela de Artes y Comunicación, se puede notar el compromiso que existe: "las competencias son mi guía al programar y diseñar las materias"; "parte de mí mismo está en los conceptos de capacitación recibida (IDP)"; "desde nuestra cosmovisión hemos trabajado por competencias desde hace mucho tiempo y por supuesto que las evaluamos"; "creo que estamos bien fundamentados". Para la Facultad de Educación y la Escuela de Música, el compromiso es evidente, ya que manifiestan: "gran parte de los docentes tiene en claro estos principios, tanto en su vida como en su cátedra"; "las creencias son el motor de la experiencia docente"; "los programas están orientados así". Al respecto, Ravela (2009), menciona que uno de los estudios más recientes y enriquecedores es el conocido como Informe McKinsey. Este estudio muestra con abundantes referencias que un buen docente hace una gran diferencia en el aprendizaje de sus estudiantes. Un mal docente, en cambio, en especial en los primeros grados de escolaridad, puede generar daños irreversibles; esto sucede cuando el docente no se compromete o no hace bien su trabajo. Para ello es necesario que exista una plana de docentes capacitados y comprometidos con el programa, donde su relación con las artes, la educación, el rigor científico debe ser congruente, como dice Taylor (2010), con una comprensión cristiana de Dios y de su creación, del origen y destino, de los principios y valores. Debemos guiar a los estudiantes a pensar profunda y espiritualmente para observar cuidadosamente y discriminar sabiamente.

La Facultad de Ingeniería y Tecnología, como ellos mismos lo manifestaron, "no estamos capacitados completamente en este aspecto", "no hay un acuerdo de cómo evaluar ni en los mismos consejos". Estas manifestaciones evidencian la falta de formación docente; por tanto, es indispensable, antes de implementar un modelo, como dice Tobón (2011), formar y aplicar la competencia docente de evaluación; es decir, pretender que los estudiantes desarrollen las competencias establecidas en el currículo obliga a los docentes a poseer también las competencias necesarias para asegurar que ello se dé. No es posible que los estudiantes formen competencias si los docentes no son, a su vez, competentes para mediar este proceso. Quiroz (2010), expresa que la aceptación e implantación de un modelo nunca es fácil. La resistencia para su aceptación se debe a que el cambio implica transformar los esquemas educati- 
vos. Un docente de la UM expresó: "El cambio no es fácil, somos tradicionales". En cuanto a la conceptualización de la evaluación del aprendizaje basado en competencias (CEVACOM), los resultados muestran que hay diversidad de opiniones y orientaciones, tal es así que para algunos docentes la evaluación debe orientarse hacia lo laboral; otros, hacia lo significativo; otros piensan que debe orientarse hacia la persona y otros, hacia los contenidos. ¿Por qué esa diversidad? ¿Hay alguna conceptualización que se ajuste al modelo? ¿Cuál es la conceptualización que maneja la UM?

Antes de dar paso a la discusión, se considera lo que manifiesta $\mathrm{Bi}$ ggs (2005), quien expresa que la evaluación es el principal complemento de la enseñanza y el aprendizaje. Si se hace mal, el resto se desmorona; de ahí la importancia de la misma, puesto que de la evaluación y de su correcta conceptualización va a depender la forma en que los estudiantes se enfrenten al proceso de enseñanza-aprendizaje. Dependiendo del significado que los docentes otorguen a la evaluación, así será la manera en que los alumnos aprendan; es decir, el significado de la evaluación condiciona la selección de estrategias para abordar el aprendizaje.

Esta declaración confirma la propuesta competencias, estrategias, desarrollo de competencias, juicios de valor, toma de decisiones, todo fundamentado en los principios, y también confirma la importancia de poseer una correcta conceptualización, tomando en cuenta la declaración expresada por uno de los docentes: "sé de profesores de otras escuelas que aún no saben lo que son las competencias, mucho menos cómo evaluar". Una realidad latente. El resultado muestra que la mayoría de los docentes de la UM piensa en una evaluación está orientada hacia lo laboral (76.00\%). Se cree que existe la idea de articular educación y sociedad con el ámbito laboral, cuya finalidad sería que los estudiantes obtengan una formación acorde con los requerimientos sociales y que, a su vez, promueva una participación más efectiva en el sector productivo. Habría que tener cuidado al asumir esta posición en la conceptualización, porque se estaría dando énfasis en el ámbito técnico o profesional solamente. Al respecto Tobón (2010), manifiesta que en esta línea se encuentran organizaciones que se enfocan únicamente en los aspectos laborales de las competencias y orientan la evaluación en torno a determinar cómo los estudiantes están en condiciones de asumir tareas técnicas, sin tener en cuenta la apropiación y movilización de saberes, como tampoco la parte humana y social del proceso. Con esta conceptualización se estarían dejando de lado los demás contextos de las competencias como el familiar, el social, el ambiental, el científico y lo espiritual, y no se estaría cumpliendo el propósito de brindar una formación integral como se establece en el Modelo Educativo 2010 de la UM. Los docentes expresaron: "la filosofía ASD está inmersa en el modelo"; "debemos plantear 
cómo hacer en nuestras próximas evaluaciones (siento, en suma, que no toma en cuenta dichos principios)".

Por otro lado, existe también la propuesta de asumir la evaluación con gran énfasis en los saberes académicos que tradicionalmente se han abordado en las instituciones educativas, porque se entiende que tener saberes conceptuales es lo mismo que ser competente. Los resultados muestran que en la UM existe un $59.75 \%$ de docentes que orientan su conceptualización hacia los contenidos. Así también lo confirman algunas declaraciones de docentes y alumnos: "es que muchos de los maestros lo ven a la antigua escuela y también lo toman siempre para rediseñar las materias y cumplir con las competencias"; "todavía se evalúa mucho a través de exámenes escritos, todavía el maestro es muy expositivo, todavía hay alto grado de autoritarismo magisterial: el maestro es la piedra, el alumno es el huevo"; "muchas clases están orientadas a la memorización solamente"; "creo que los profesores en ocasiones no tienen una buena manera de evaluar, deberían ser precisos en la forma en la que evalúan"; "Ios maestros se enfocan demasiado en los exámenes y no usan otros métodos como las presentaciones orales o visuales para ayudar en la comprensión de las materias. Es decepcionante". Frente a esta realidad, ¿qué debemos hacer?

Tobón (2010), aconseja en esta perspectiva y se plantea que el enfoque del trabajo docente debe ir hacia los contenidos disciplinares, pero con participación activa de los estudiantes. Entonces, la evaluación de competencias sigue llevándose a cabo sobre contenidos, aunque con cambio en la forma. Una persona es competente si posee conocimientos, destrezas y aptitudes para desempeñarse en una situación determinada.

Para finalizar esta segunda discusión sobre conceptualización, las preguntas planteadas al inicio, sobre si existía alguna conceptualización que se ajuste al modelo y lo que plantea la UM, las pruebas realizadas a través del modelo de ecuaciones estructurales (SEM) para ver cuáles son los mejores predictores entre CEVACOM y GaMoTeC, se encontró que las variables que más aportan para la relación de la variable CEVACOM fueron: evaluación orientada hacia la persona y evaluación orientada hacia lo significativo, ¿serán estas orientaciones las que se ajustan a nuestro modelo?

Al comparar el concepto de competencias que maneja la Universidad de Montemorelos (2010), como conjunto de habilidades para transferir el conocimiento con una actitud que va más allá de la sola resolución de problemas, a la consecución de los objetivos en el cumplimiento de una misión. Aunque no maneja un concepto en evaluación; sin embargo, nos da la idea de formación integral orientada hacia la persona y que sea un proceso significativo.

El modelo que se propone inicia con esta conceptualización, donde además se integran lo laboral y los contenidos, como lo muestra el análisis rea- 
lizado a través de conglomerados de k medias, una marcada homogeneización en sus características con énfasis en lo significativo, lo laboral, el contenido y hacia la persona. En la UM, 48 docentes tienen esta orientación y con el resto, ¿qué pasó? Los docentes y alumnos expresaron: "hay conceptos que faltan por definir"; "Cada docente, muchas veces, se enfoca en su propia materia y no evalúa todo el perfil que debe adquirir el estudiante"; "más balance entre los componentes. Las actitudes, las habilidades y los conocimientos: saber, saber hacer y saber ser, ya que puede ser que los maestros enfoquen más los aspectos actitudinales y no los aspectos de dominio conceptual, prácticoprofesional. Más de la práctica"; "le falta un enfoque más marcado para tener las competencias al 100\%"; "se busca que las competencias se hagan con una cosmovisión cristiana".

Por último, el tercer punto a discutir es en relación con la estimulación del aprendizaje a través de las evaluaciones practicadas en la UM. Los docentes perciben que la estimulación es alta o mayor mientras que los estudiantes la perciben baja o menor; sin embargo, ambos están de acuerdo en que existe estimulación. Además, esas estimulaciones proporcionan al estudiante beneficios como también inseguridades. Tanto las pruebas estadísticas descriptivas como la prueba estadística t de Student para muestras independientes así lo confirman. ¿Por qué esas diferencias de percepción?

Según investigaciones, los docentes tienen una mejor percepción sobre el estímulo que ejerce la evaluación en el aprendizaje de los alumnos, cuando aprenden a cultivar ciertas características peculiares, tales como conocer al alumno, interrelacionarse con él (Arévalo, 2010), de tal manera que la evaluación sea precisa, justa y veraz. Esteban (2002), sostiene que los docentes poseen, efectivamente, un marco teórico personal para explicar aquello que observan y deciden cotidianamente. Todo sistema de evaluación debe perseguir un objetivo prioritario que no es otro que el alumno aprenda, más concretamente, que el alumno aprenda a aprender, aprenda a autogestionar el aprendizaje (Álvarez y Villardón, 2006; Biggs, 2005) o, como señalan Hernández, Martínez, Da Fonseca y Rubio (2005), un aprendizaje que sirve para construir un cuerpo de conocimientos, habilidades o destrezas y actitudes flexibles, autoestructurantes, y que le permitan seguir aprendiendo autónomamente a lo largo de toda su vida. Si los docentes conocen esas variables y la forma en que pueden influir en el aprendizaje, podrán mejorar su intervención en dicho proceso modificando, si fuera oportuno, la metodología docente, el proceso de enseñanza, el tipo de evaluación y las técnicas a emplear; de esta manera podrían contribuir mejor a que el alumno construya su propio aprendizaje.

En cuanto al alumno, el grado de estimulación del aprendizaje que resultó media unidad más bajo $(M=3.54, D E=.509)$ que el del docente, se 
debe a muchos factores, porque el alumno, como menciona Díaz (2003), es una realidad compleja y sumamente variable. Díaz observó que muchos de los estudiantes estudian para aprobar los exámenes; otros, para adquirir solo información; algunos son motivados a la fuerza, mientras que para otros su reacción es innata: les gusta estudiar porque están motivados. Agrega que la evaluación debe ser una ayuda efectiva del aprendizaje y que para que esto suceda, es necesario que cambie la situación descrita y que la evaluación respete la dignidad y el valor de todos los estudiantes. Es necesario también hacer que los alumnos se sientan más seguros de sí mismos y promover respuestas emocionalmente deseables, a fin de que les proporcione estabilidad emocional. De la Orden (1978, citado en Díaz, 2003), sostiene que uno de los objetivos prioritarios de los estudiantes, en general, es satisfacer las exigencias de los exámenes; todo profesor conoce la capacidad de los alumnos para percibir las características, hábitos y peculiaridades evaluadoras de los docentes (temas con mayor probabilidad de salir, tipo de cuestiones, forma de calificar) y para transformar en las metas reales de su aprendizaje los conocimientos y conductas que les permitan superar con éxito las pruebas.

Rosario et al. (2008), encontraron que el género está relacionado con el modo en que se interpreta y responde a diversas situaciones de evaluación académica. Los chicos tienden a afrontar las situaciones de examen como un desafío, implicándose más en la tarea cuanto mayor sea la competencia percibida para superarla. Sin embargo, las chicas, por cuestiones asociadas a exigencias sociales que enfatizan la necesidad de implicarse y responsabilizarse en las tareas, perciben las situaciones de examen como más amenazadoras, lo que les provoca comportamientos ansiosos.

Amato y Novales (2009), encontraron una tendencia estadísticamente significativa con una asociación baja. Los estudiantes mostraron acuerdo con la afirmación de que las actividades de evaluación entre pares les parecían justas y formativas (70\%) y con la percepción de un mejor aprendizaje.

Asimismo, el GaMoTeC explica el $28.6 \%$ de la variación GEAEV, encontrando que sí existe relación significativa entre las variables y son dos dimensiones, PriOPR y EstCC, las predictoras.

Lógicamente, si no se cuenta con un buen modelo de evaluación de las competencias no se pueden desarrollar las competencias y menos habría aprendizaje. La enseñanza, el aprendizaje y la evaluación deben constituirse en una unidad indisoluble (Coll, Martín y Onrubia, 2001), de manera tal que la evaluación sirva para explicar tanto los aprendizajes de los estudiantes como la actividad instruccional del maestro. La evaluación, entonces, servirá para ofrecer información a los estudiantes acerca de los aprendizajes alcanzados, a fin de que puedan asumir la responsabilidad de controlar sus logros. En otras 
palabras, ofrecerá a los actores del hecho educativo información útil y relevante para mejorar cada uno su acción: a los alumnos, para que los conocimientos construidos sean más amplios, significativos y profundos, y a los profesores, para que su actividad didáctico-pedagógica sea más eficaz en cuanto a promover esos mejores aprendizajes (Esquivel, 2009). Sadler (1989) indicó que no es suficiente que los maestros simplemente señalen si las respuestas dadas en una prueba son correctas o incorrectas, o si la tarea ejecutada (o el producto) exhibido refleja aprendizajes significativos. Esta realimentación deberá ir necesariamente acompañada de criterios explícitos y claros de desempeño, así como de información a los estudiantes sobre estrategias para facilitar el aprendizaje. Este aporte de Sadler se acerca a la conceptualización aportada por Coll, Martín y Onrubia (2001), según la cual la información que ofrece el juicio de valor deberá ayudar al profesor a tomar decisiones que mejoren sus actividades de enseñanza y a los estudiantes, a mejorar su aprendizaje. Esta función de regulación de los procesos de enseñanza y de aprendizaje hace que se la denomine evaluación reguladora. Por otra parte, según señalan los autores citados arriba, "se ha subrayado su vertiente formadora, es decir, su utilidad para que los alumnos aprendan a regular sus procesos de aprendizaje" (p. 5).

\section{Conclusión}

Las conclusiones que afloran de esta investigación son las siguientes:

1. Se logró determinar que sí existe aceptación del modelo teórico de evaluación del aprendizaje basado en competencias (GaMoTeC) en una proporción medianamente aceptada (75.25\%).

2. Entre las facultades y escuelas, las diferencias son significativas, en cuanto a la aceptación del modelo, encontrándose los niveles más altos de aceptación en la Escuela de Artes y Comunicación, en la Facultad de Educación y en la Escuela de Música; y las que menos aceptan el modelo fueron la escuela de preparatoria y la Facultad de Ingeniería y Tecnología.

3. La aceptación de cada elemento estructurante del modelo se refleja a través de las medias, que son altas para principios y procesos, y un poco más bajas para estrategias.

4. La conceptualización de la evaluación de las competencias en la UM es diversificada y tiene diferentes orientaciones, predominando el concepto de evaluación orientada hacia lo laboral (76\%), el 73.75\% hacia lo significativo, un $67.75 \%$ hacia la persona y un $59.75 \%$ hacia los contenidos.

5. Considerando la teoría de los enfoques, 14 administradores y docentes de la UM orientan su conceptualización hacia el enfoque funcionalista (muy marcado en lo laboral, lo significativo y los contenidos; menor en la persona); 
43 orientan su conceptualización en el enfoque conductual-organizacional (muy marcado en lo laboral, lo significativo y lo personal; menor en contenidos); 59 orientan su conceptualización hacia el enfoque constructivista (homogéneo en lo laboral, lo significativo, lo personal y los contenidos) y 48 orientan su percepción hacia el enfoque socioformativo (muy homogéneo en lo significativo, lo laboral, los contenidos y hacia la persona).

6 . La relación entre las variables CEVACOM y GaMoTeC es directa medianamente fuerte $(\phi=.54)$, siendo las variables predictoras para el CEVACOM la evaluación orientada hacia la persona $(\lambda=.66)$ y la evaluación orientada hacia lo significativo $(\lambda=.63)$; y para la variable GaMoTeC, los elementos procesos $(\lambda=.93)$ y el elemento principios $(\lambda=.92)$.

7. La evaluación orientada hacia la persona $(\lambda=.66)$ y la evaluación orientada hacia lo significativo $(\lambda=.63)$, equilibradas con la evaluación orientada hacia lo laboral y hacia los contenidos, representa el concepto percibido por los docentes de la UM.

8. Para los administradores y docentes, el grado de estimulación del aprendizaje influido por las evaluaciones es alto $(M=4.05, D E=0.391)$ mientras que para los estudiantes también es alto pero con media unidad más baja $(M=3.54, D E=0.509)$.

9. Existe diferencia entre las medias de la variable GEAEV según la percepción de docentes y estudiantes, siendo la percepción mayor en docentes y menor en estudiantes. Podemos afirmar que la evaluación de las competencias es una ayuda del aprendizaje y desarrollo de las competencias.

10. Las dimensiones del GaMoTeC predicen al GEAEV, explicándolo en un $\mathbf{2 8 . 6 \%}$ de su variación, existiendo relación significativa entre las variables $(F=33.58, g l .=161, p=.000)$.

11. Las dos dimensiones del GaMoTeC que explican de manera significativa a la variable GEAEV son: la dimensión PriOPR $(\beta=0.453)$ que es la más importante, pues explica tres veces más y la que le sigue es la dimensión $\operatorname{EstCC}(\beta=0.150)$.

12. En cuanto a las creencias de los administradores y docentes con respecto a las prácticas de evaluación por competencias en la UM, el 76.2\% considera que sí se están llevando a cabo, y el $23.8 \%$ cree que no; asimismo, el $75.6 \%$ cree que las estrategias que se están usando en la evaluación de las competencias en la UM son las adecuadas, y el $24.4 \%$ cree que no. Para los procesos, el $79.9 \%$ cree que estos apoyan al desarrollo de las competencias, y el $20.1 \%$ cree que no; y por último, el $95.1 \%$ de los docentes creen que las evaluaciones de las competencias en la UM toman en cuenta los principios filosóficos de la educación adventista, y el $4.9 \%$ no creen en esa posibilidad. 
13. Acerca de las estrategias de evaluación que los docentes utilizan en sus clases, según los estudiantes, son: el portafolio (82.7\%), la investigación $(77.2 \%)$, los proyectos $(73.2 \%)$ y la resolución de problemas (68.1\%). Las estrategias que menos usan son los registros de incidentes críticos, las escalas de valoración, las listas de control, los debates, la evaluación por pares o coevaluación y las pruebas de desarrollo.

14. Las experiencias vividas por los estudiantes en cuanto a la evaluación del aprendizaje basado en competencias giran en torno a dos grandes grupos: experiencias positivas y experiencias negativas, las mismas que han sido clasificadas en diversas categorías. Entre las más resaltantes se encuentran: "Este modelo por competencias me obliga a poner mi esfuerzo, porque al final debo ser competente para algo específico, me obliga a razonar y a pensar inteligentemente aplicando mis conocimientos en casi todas las materias. Para mí este modelo sí ayuda"; "creo que es muy interesante, debido a todos los métodos de evaluación que se tiene, y gracias a eso, siento que he aprendido más"; "todavía no comprendo este sistema exactamente qué es lo que quieren, pero en lo que sí estoy seguro es que es una buena manera de aprender y desarrollar mi intelecto"; "creo que los profesores en ocasiones no tienen una buena manera de evaluar, deberían ser precisos en la forma en la que evalúan"; "casi nunca evalúan de acuerdo a competencias, y si lo hacen no nos notifican las competencias a evaluar"; y por último, "pues me he dado cuenta de lo que soy capaz de hacer por mí y por los demás. Ha sido buena".

15. El Modelo teórico de evaluación del aprendizaje basado en competencias (MOTEC) en base a los índices de bondad de ajuste, presenta una relación significativa $(p<0.05)$ entre CEVACOM y GaMoTeC, siendo la bondad de ajuste alta en el CFI (0.977), aceptable en el RMSEA (0.073) y significativa con respecto al ajuste global, según la Chi-cuadrada $\left(C h i^{2}=24.276, p=.029\right)$.

\section{Recomendaciones}

1. Se recomienda considerar que, una vez realizado este trabajo, han quedado una serie de cuestiones planteadas para su resolución en trabajos posteriores y que, por lo tanto, se deben abrir nuevas líneas de investigación futuras.

2. Realizar estudios sobre la necesidad de evaluar competencias del saber, del saber hacer y del saber ser; para ello, es necesario incorporar nuevos instrumentos de evaluación, válidos a cada tipo de resultado de aprendizaje, y comprobar su eficacia y eficiencia.

3. Hacer investigaciones en relación a las potencialidades de la tecnología en la evaluación de competencias como herramienta de apoyo útil para este fin. 
4. Efectuar estudios que permitan incorporar distintos instrumentos de evaluación que ayuden al alumno durante el proceso de aprendizaje a consolidarlo, aplicarlo y hacerlo suyo.

5. Llevar a cabo estudios sobre cómo combinar la autoevaluación con técnicas de coevaluación, responsabilizando a los estudiantes como sujetos y agentes de su propia evaluación.

6. Realizar estudios sobre evaluación de las competencias en educación superior a través del portafolio, entre otros.

Haciendo una valoración global y sintética de este artículo, se cree que se ha contribuido, en primer lugar, a mostrar una visión profunda acerca de una serie de posiciones teóricas de máxima relevancia sobre el tema de la evaluación de las competencias. En segundo lugar, al utilizar conjuntamente variables de los estudiantes y docentes, y cruzar la información con los predictores del GaMoTeC, se ha ido más allá de los habituales trabajos sobre el tema. En tercer lugar, la propuesta del modelo teórico de evaluación del aprendizaje basado en competencias (MOTEC) y su validación empírica a través de su aceptación son un intento formal para conducir y mostrar el camino a los futuros trabajos que pretendan explicar la evaluación de las competencias. En cuarto lugar, la profundidad teórica expuesta y la complejidad metodológica en el análisis de los datos para abordar la incidencia que tienen los elementos y factores que intervienen en el modelo y las variables estudiadas constituye un aporte actualizado y sustantivo, que permite una aproximación científica y exacta al tema en cuestión. Quinto lugar, el uso de los programas informáticos para medir el ajuste de este modelo es un aporte importante, tanto en la validación del constructo, como en la confianza sobre la estimación y el ajuste que ofrece AMOS.

Finalmente, en el aspecto personal, el conocimiento en profundidad de este tema tan relevante ha trasformado la manera de ver el mundo de parte del investigador, a los estudiantes y a los docentes. La complejidad del tema, los múltiples y contrarios pensamientos, la tan extensa literatura y el aprendizaje de una metodología muy compleja, actuaron como reactivo interno para que se dedique plenamente, día a día, a un trabajo laborioso, a veces lento, solitario y cansado, pero intensamente enriquecedor. 


\section{Referencias}

Álvarez, Y. y Villardón, L. (2006). Planificar desde competencias para promover el aprendizaje. El reto de la sociedad del conocimiento para el profesorado universitario. Cuadernos monográficos del ICE, 12. Bilbao: Universidad de Deusto.

Amato, D. y Novales, X. de J. (2009). Aceptación del aprendizaje basado en problemas y de la evaluación entre pares por los estudiantes de medicina. Gaceta Médica de México, 145(3), 197-205.

Arévalo, R. M. (2010). Teorías de dominio de los docentes sobre el aprendizaje y su expresión en la evaluación de los aprendizajes: un estudio de caso en una institución educativa particular de Lima. Educación, 19(36), 23-42.

Biddle, B., Good, T. y Goodson, I. (2000). El contexto social cambiante. Implicaciones para los profesores y para la enseñanza. En B. Biddle, T. Good, y I. Goodson (Comp.), La enseñanza y los profesores III. La reforma de la enseñanza en un mundo de transformación (pp. 77-94). Barcelona: Paidós.

Biggs, J. (2005). Calidad del aprendizaje universitario. Madrid: Narcea.

Clark, B. (2000). Creando universidades innovadoras, estrategias organizacionales para la transformación. México: UNAM-Porrúa.

Coll, C., Martín, E. y Onrubia, J. (2001). La evaluación del aprendizaje escolar, dimensiones psicológicas, pedagógicas y sociales. En C. Coll, J. Palacios y A. Marchesi, Desarrollo psicológico y educación. Psicología de la Educación (Vol. 2, pp. 549-567). Madrid: Alianza.

Coronado, M., Sánchez, A., Vega y León, S. y Jiménez, Ma. del R. (2001). Construcción de competencias laborales a partir de los currícula universitarios. Argumentos, 38, 91 122.

Cronbach, L. J. (1963). Course improvement through evaluation. Teachers College Record, 64, 672-686.

Danielson, Ch. y Abrutyn, L. (2002). Una introducción al uso de portafolios en el aula. México: Fondo de Cultura Económica.

Declaración sobre la filosofía adventista de la educación. (2002). Revista de Educación Adventista, $15,15-18$.

De la Orden, A. (1982). La evaluación educativa. Buenos Aires: Docencia.

Díaz, M. (2003). Las evaluaciones escritas y la actitud de los alumnos frente al estudio. Tesis de maestría, Universidad Peruana Unión, Lima, Perú.

Esquivel, J. M. (2009). Evaluación de los aprendizajes en el aula: una conceptualización renovada. En E. Martín y F. Martínez Rizo (Coord.), Avances y desafíos en la evaluación educativa (pp. 127-144). Madrid: OEI. Fundación Santillana. 
Esquivel, V. (2006, octubre). De la integración a la educación inclusiva. Educación para todos. Conferencia presentada en el congreso anual de la ANDE, San José, Costa Rica.

Esteban, M. (2002). Acerca del conocimiento del mundo, de los fenómenos físicos y sociales, de nuestros contemporáneos y amigos de nosotros mismos. RED. Revista de Educación a Distancia, 2, 1-5. Recuperado de http://www.um.es/ ead/red/2/teoriasimplicitas. pdf

Grajales, T. (2008). Cómo planear una investigación empírica. Una experiencia de autoaprendizaje. Montemorelos: Publicaciones Universidad de Montemorelos.

Hernández, F., Martínez, P., Da Fonseca, P. S. L. y Rubio, M. (2005). Aprendizaje, competencias y rendimiento en educación superior. Madrid: La Muralla.

Hernández Sampieri, R., Fernández Collado, C. y Baptista Lucio, P. (2006). Metodología de la investigación. México: McGraw Hill.

Lluch, E. (1998). Introducción a la educación basada en competencias. Educación: Revista del Consejo Nacional Técnico de la Educación, 53, 81-104.

Matos Chamorro, A. (2008). Enseñanza-aprendizaje y evaluación por competencias en carreras de ingeniería. Revista Internacional de Estudios en Educación, 8(2), 71-82.

Moreno, P. (2000). La transición del modelo educativo cerrado al abierto en América Latina: el caso de la educación por competencias en México. En I Congreso Internacional de Educación, crisis y utopías (pp. 159-166). Buenos Aires: Universidad de Buenos Aires.

Pimienta, J. H. (2008). Evaluación de los aprendizajes. Un enfoque basado en competencias. México: Pearson-Prentice Hall.

Pimienta, J. y Enríquez, A. (2009). Educación basada en competencias. Guía para la aplicación del enfoque. México: Pearson.

Quiroz, M. E. (2010). Modelos educativos en el IPN y el ITESM. Las competencias profesionales en la educación superior. México: ANUIES.

Ravela, P. (2009). La evaluación del desempeño docente para el desarrollo de las competencias profesionales. En E. Martín y F. Martínez Rizo (Coord.), Avances y desafíos en la evaluación educativa (pp. 113-126). Madrid: Santillana.

Real Academia Española. (2001). Diccionario de la Real Academia Española (22 Ed.). [Versión electrónica]. Recuperado de http://www.rae.es

Reynaga, S. (2003). Educación, trabajo, ciencia y tecnología. México: Consejo Mexicano de investigación educativa, SEP-CESU.

Rosario, P., Nuñez, J. C., Salgado, A., González Pienda, J. A., Valle, A., Joly, C. y Bernardo, A. (2008). Ansiedad ante los exámenes: relación con variables personales y familiares. Psicothema, 20(4), 563-570.

Sadler, R. (1989). Formative Assessment and the Design of Instructional Assessment. Instructional Science, 18, 118-144. 
Moisés Díaz Pinedo

Scriven, M. (1967). The Methodology of Evaluation. En R. W. Tyler, R. M. Gagne y M. Scriven (Eds.), Perspectives of curriculum evaluation (pp. 39-83). Chicago: Rand McNally.

Stake, R. E. (1983). La evaluación de programas; en especial la evaluación de réplica. En W. B. Dockrell y D. Hamilton (Eds.), Nuevas reflexiones sobre la investigación educativa (pp. 91-108). Madrid: Morata.

Stufflebeam, D. (1987). Evaluación sistemática. Madrid: Paidós.

Stufflebeam, D. y Shinkfield, A. J. (2002). Evaluación sistemática: Guía teórica y práctica. Barcelona: Paidós Ibérica.

Taylor, J. W. (2010). Un enfoque cristiano para las artes. Revista de Educación Adventista, 30, 18-22.

Tobón, S. (2007). Metodología general de diseño curricular por competencias desde el marco complejo. Recuperado de http://www.cife.ws

Tobón, S. (2009). Formación basada en competencias: pensamiento complejo, diseño curricular y didáctico. Bogotá: ECOE.

Tobón, S. (2010). Formación integral y competencias. Pensamiento complejo, currículo, didáctica y evaluación. Bogotá: Ecoe Ediciones.

Tobón, S. (2011). Evaluación de las competencias en la Educación Básica. México: Santillana.

Tobón, S., Pimienta, J. H. y García, J. A. (2010). Secuencias didácticas: Aprendizaje y evaluación de competencias. México: Pearson Educación.

Tyler, R. (1949). Basic Principles of Curriculum and Instruction. Chicago: University of Chicago.

Universidad de Montemorelos. (2010). Compromiso educativo 2006-2011. Modelo Educativo 2010. El desarrollo de la docencia. Montemorelos, Nuevo León, México: Vicerrectoría Académica.

White, E. (1965). Ministerio de curación. Mountain View: Publicaciones Interamericanas.

White, E. (1975). La educación cristiana. Buenos Aires: Asociación Casa Editora Sudamericana.

White, E. (1978). La educación. Florida, Buenos Aires: Asociación Casa Editora Sudamericana.

White, E. (2005). Consejos para los maestros, padres y alumnos. México: APIA.

Woodruffe, Ch. (1993). What is Meant By a Competency? Leadership and organization. Development Journal, 14(1), 29-36. 